\title{
Visual Information Management Based on Visual Communication of Deep Learning
}

\author{
Wenting Wang $\mathbb{D}^{1,2}$ and Younghwan Pan $\mathbb{D}^{1}$ \\ ${ }^{1}$ Interaction Design Lab, Graduate School of Techno Design, Kookmin University, 861-1 Jeongneung-dong, Seongbuk-gu, \\ Seoul 02707, Republic of Korea \\ ${ }^{2}$ School of Fine Arts and Design, Chengdu University, 2025 Chengluo Avenue, Chengdu 610106, China \\ Correspondence should be addressed to Younghwan Pan; peterpan@kookmin.ac.kr
}

Received 11 October 2021; Revised 6 December 2021; Accepted 11 December 2021; Published 21 January 2022

Academic Editor: Rahman Ali

Copyright (C) 2022 Wenting Wang and Younghwan Pan. This is an open access article distributed under the Creative Commons Attribution License, which permits unrestricted use, distribution, and reproduction in any medium, provided the original work is properly cited.

\begin{abstract}
To speed up the buffering of visualization images and ensure the integrity of visualization images, this paper proposes the construction of a virtual packaging model of visualization images based on visual communication of deep learning (DL). Based on the visual communication, the specific calibration steps of the visual image are designed to complete the adjustment of the visual image by using the calibration process of the visual image. We introduce the minimum buffer coefficient of the visual image, calculate the size of the buffer liner of the visual image, correct the thickness of the visual image, and complete the design of the buffer package of the visual image. Finally, the process of constructing the virtual packaging model of the visualized image is constructed to realize the virtual packaging of the visualized image. The simulation results show that the visual image virtual packaging model based on visual communication improves the buffering speed of visual image by $66.6 \%$ compared with the visual image virtual packaging model based on machine vision.
\end{abstract}

\section{Introduction}

With the rapid development of science and technology, visualization images are widely used in industries such as automotive maintenance, aerospace, food packaging, logistics consignment, and packaging manufacturing $[1,2]$. Visualization image technology occupies a major position in the production of modern large-scale enterprise life. Although visualization image technology is gradually improving in language and component settings, the old system inside the application image technology still involves a series of integration problems. Visual image technology is required to hide the internal structure of legacy old systems when analyzing objects, create a good port to the external world, and finally output a unified description and transmission of data. Although visual image techniques can reduce the processing time of legacy old systems, there are still various unknowable pitfalls of visual image techniques when applied in practice [3]. Hence, the research and development of visual image virtual packaging model constructs that combine the advantages of multiple technologies are a necessary topic without delay.

The key contribution of this paper includes the following:

(i) This paper presents the virtual packaging model construction of visualization images based on visual communication of DL. By using the calibration procedure of the visual image, the specific calibration steps of visual image are considered to complete the calibration of the visual image, the minimum buffer coefficient of the visual image is introduced, the size of buffer liner of visual image is calculated, and the design of buffer packaging of the visual image is accomplished by adjusting the thickness of visual image. 
(ii) Finally, the virtual packaging model construction process of the visual image is realized through the virtual packaging.

(iii) The simulation results show that the visual image virtual packaging model based on visual communication can accelerate the buffering speed of the visual image.

\section{Design of Virtual Packaging Model Construction Technique for Visualized Images}

2.1. Calibration of Visual Images. Visual image calibration is the process of calibrating the entire operation by taking pictures or continuous video using the camera or camcorder that comes with the system itself [4]. For visualization images, image calibration visual system, labeling position relationship, and other links need to use the virtual packaging model construction technology and must strictly operate the relevant links before and after the order. Figure 1 can be used to accurately determine the reading visualization image calibration process.

The whole process of the calibration of the visualized image is step-wise discussed as follows:

Step 1: define the scale factor of the visual image virtual packaging construction model, first defining the scale factor of the conveyor belt [5]. The definition of scale factor is to use the encoder on the conveyor belt to record that the target object will be rotated after moving a certain distance, generating a relative incremental angle, and then the change in the relative incremental angle of the rotation is converted into the actual distance of the target moving on the conveyor belt [6]. Relative to the same moving target object, if the encoder angle of the conveyor belt needs to remain fixed and the value cannot change, then for the visualized image, the encoded values of the feature points after moving the target object at different distances need to be measured several times and the scale factor is calculated to make the data more accurate $[7,8]$.

Step 2: the geometric calibration of the conveyor belt by the visual image virtual packaging model is determined by the dynamic coordinate system of the working area on the conveyor belt. The calibration of the working area needs to consider the width of the belt, the installation position of the belt, the maximum workload of the belt itself, and other factors.

Step 3: through the visual image virtual packaging model calibrated by the camera, we use the camera to capture the position of the calibration plate and use the system with the automatic recognition system to capture all feature points. Finally, we calibrate the grid through the calibration tool of the software itself. The calibration of visual coordinates and tool coordinate system is based on the completion of the above operation. During the conversion and calibration, the grid cardboard is kept still, the conveyor belt is started, and

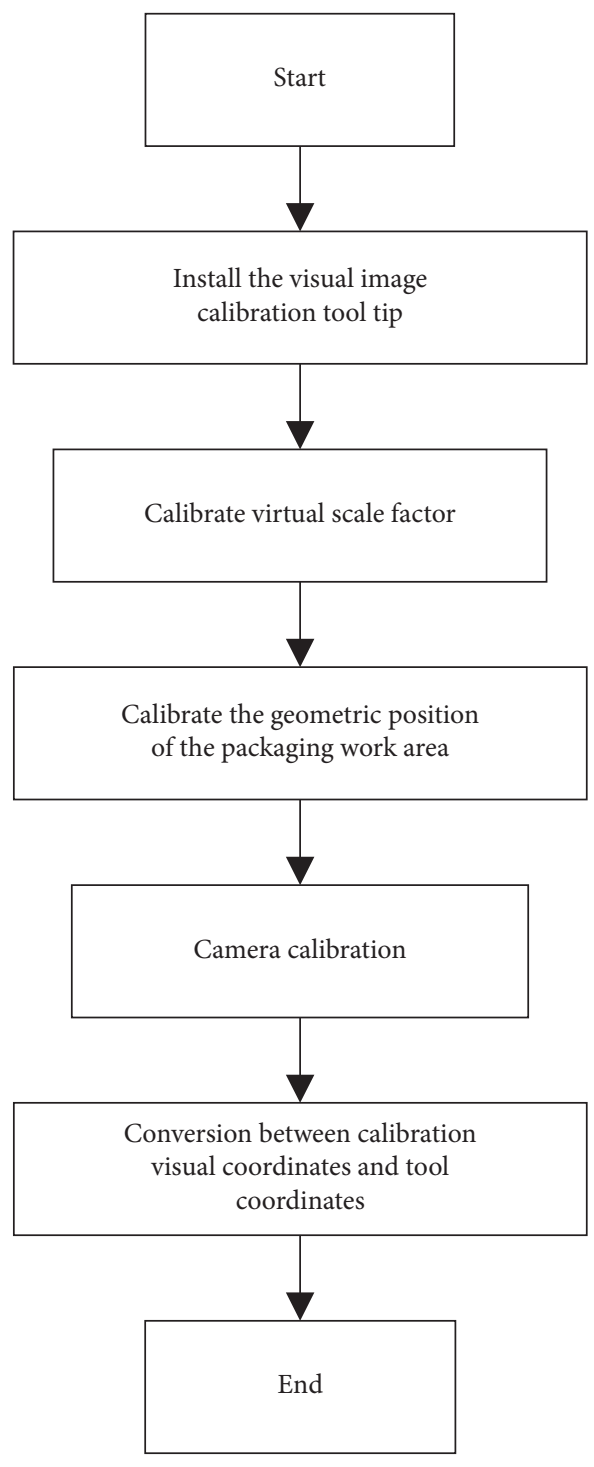

Figure 1: Flow chart of visual image calibration process.

the moving target is transferred to the working area set in Step 2 and then moved to the previously selected coordinate system origin and the $X Y$ direction of the coordinate axes; the grid cardboard is used to record the moving target's origin and record the trajectory in the $X$ direction and $Y$ direction.

2.2. Designing Visual Image Cushioning Packages. Since the visualization image cannot complete the virtual packaging task naturally, in order to speed up the buffering of the visualization image, designing the visualization image buffer packaging can ensure the integrity of the visualization image. The visualization image has a wide range of applications in virtual packaging, and in order to avoid electrostatic damage to the visualization image, the projector is first wrapped with an antistatic bag to calculate the size of the visualization image buffer liner based on the minimum buffer coefficient of the visualization image [9]. The cushion size diagram is shown in Figure 2. 


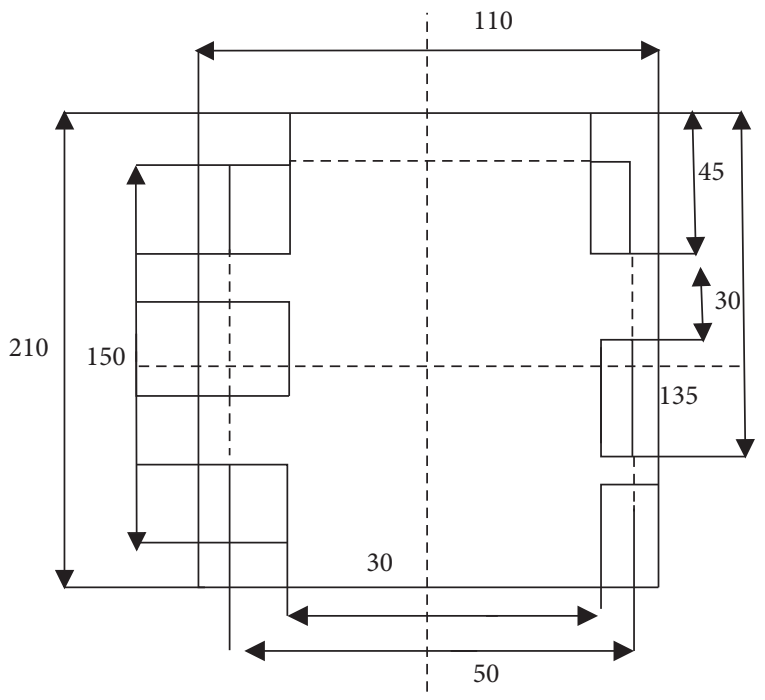

Figure 2: Dimensional drawing of the liner.

The minimum buffering factor for visualized images allows the visualized image to use the least amount of material for the same buffering effect. Equation (1) can be used for the thickness and area of the visual image buffer liner:

$$
\begin{gathered}
t=\frac{C H}{G}, \\
A=\frac{G W}{\sigma_{m}},
\end{gathered}
$$

where $t$ denotes the cushion thickness of the visual image; $C$ denotes the cushioning factor of the visual image; $H$ denotes the drop height of the visual image with the same cushioning effect; $A$ denotes the cushioned cushion area of the visual image; $G$ denotes the fragility value of the visual image; $W$ denotes the image size; and $\sigma_{m}$ denotes the maximum stress of the visual image.

\subsection{Building Virtual Packaging Models for Visual Images.} An image packing model is used to calculate the moving trajectory of the visualized image. A multiangle geometric cropping and shearing are used to solve the objective problem and the robustness problem due to print scanning, a colorful visualized image embedding capacity, and print scanning resistant visualized image method $[10,11]$. The construction process of the virtual packaging model for visualized images is as follows:

(1) According to the visual communication coding rules, the carrier information and background information of the visual image are separated into the two-dimensional code shading area of the visual image [12].

(2) The frequency response after predistortion processing of visual image is calculated by using

$$
W^{\prime}(\omega)=\partial \cdot W(\omega) \cdot \frac{1}{P(\omega)},
$$

where $W^{\prime}(\omega)$ is the frequency response after visual image predistortion processing; $W(\omega)$ is the original frequency response of the visual image; $\partial$ is the compensation coefficient; and $P(\omega)$ is the visual image shock frequency response.

(3) The visual communication is implemented on the visual image, and the background sequence is inserted to obtain the encrypted background sequence.

(4) The visual color shading image extracted in Step 1 is transformed in color space to extract the brightness component of the transformed visual image [12].

(5) The luminance component of the visual image obtained in Step 4 is transformed by visual communication and optimized the obtained packaging coefficient.

(6) The transformed packaging coefficient is decomposed and transformed by visual communication. Using the rules of visual communication, the background information of the visual image is embedded into the decomposed diagonal matrix. The embedded background information is located at 300 pixels of the diagonal matrix to obtain the diagonal matrix containing background information $[13,14]$.

(7) The diagonal matrix of the decomposed visual image is inverse decomposed to obtain the brightness component matrix containing image background information.

(8) The luminance component matrix containing image background information is converted in the color space to obtain the visual image of color shading carrier containing background information. The image shading information and two-dimensional code information are combined in PS software to visually package the visual image.

\section{Proposed Approach and Result Analysis}

In this section, the proposed approach and result are discussed by chart representation.

3.1. Simulation Analysis. During the experiment, 10 groups of buffer condition data of visual images are prepared for simulation analysis. To ensure the objectivity of simulation analysis, the simulation buffer environment is divided into 10 experimental groups including 3 simulation levels. Using the visual image virtual packaging model based on machine vision as the experimental comparison object, we carried out accelerated simulation experiments on the visual image buffer. In Figure 3, the simulation environment is shown.

During the experiment, two computers with the same configuration were prepared, the simulation software was installed and run, and the simulation data of Figure 3 were loaded into the simulation program, and two no-load experiments were conducted before the experiment started to ensure the stability of the experimental data interface. Then, 


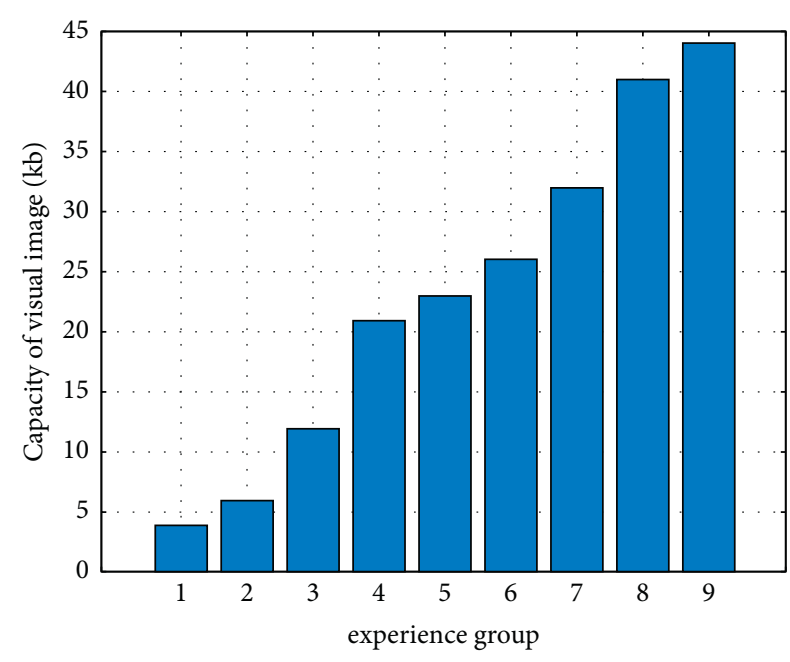

Figure 3: Simulation environment.

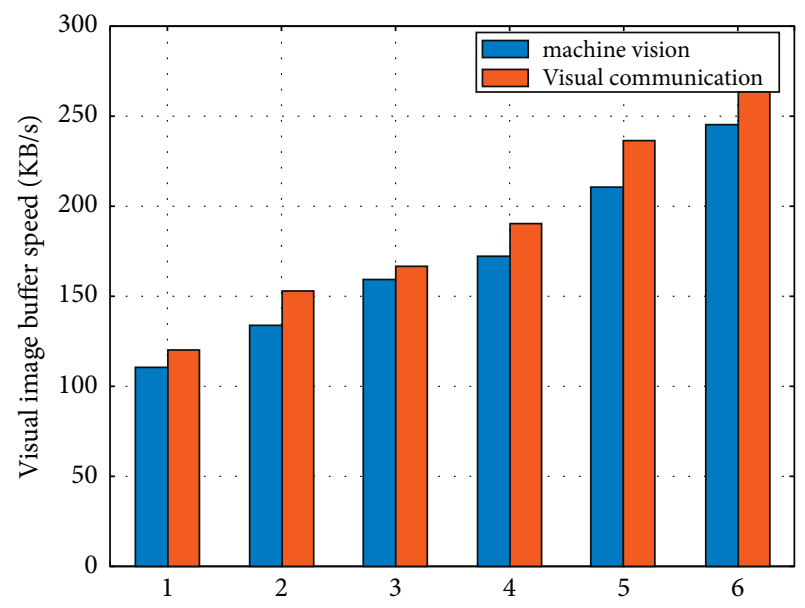

Figure 4: Comparison of visual image buffering speed.

according to the data interface of the simulation software, the visualization image virtual packaging model based on visual communication and the visualization image virtual packaging model based on machine vision are loaded into the simulation program, and the visualization image buffer was prepared to speed the simulation experiment. In Figure 4, a comparison of the visual image buffering speed is plotted by using visual image buffering speed obtained from the two visual image virtual packaging models.

From the experimental results, the virtual packaging model of visual images based on machine vision is used to virtually package the visual images; the buffering speed of the visual images is slow as the capacity of the visual images gradually increases. The buffering speed of the visualized image is slower as the capacity of the visualized image increases. Hence, the trend of slowing down the buffering speed is small, and the overall buffering speed is still faster. The average buffering speed of the visualized image is $72.8 \mathrm{~Kb} / \mathrm{s}$ in the 10 experiments, which is $66.6 \%$ higher than the buffering speed of the virtual packaging model of the visualized image using machine vision.

\section{Conclusions}

This paper proposes a virtual packaging model construction of visualization images based on visual communication. Using the calibration process of visual image, the specific calibration steps of the visual image are designed to complete the calibration of the visual image. The minimum buffer coefficient of the visual image is introduced, the size of buffer liner of visual image is calculated, and the design of buffer packaging of visual image is completed by correcting the thickness of visual image. Finally, the virtual packaging model construction process of visual image is realized through the virtual packaging. The simulation results show that the visual image virtual packaging model based on visual communication can accelerate the buffering speed of visual image.

\section{Data Availability}

The data used to support the findings of this study are included within the article.

\section{Conflicts of Interest}

The authors declare that there are no conflicts of interest regarding the publication of this paper.

\section{References}

[1] Y. Yamanouchi, H. Mitsumine, S. Inoue et al., "Construction of omnidirectional images for image-based virtual studio," Visual Communications \& Image Processing. International Society for Optics and Photonics, vol. 4067, pp. 1517-1524, 2000.

[2] C. Ji, G. Duan, H. Ma, L. Zhang, and H. Xu, "Modeling of image, video and text fusion quality data packet system for aerospace complex products based on business intelligence," Journal of Visual Communication and Image Representation, vol. 59, no. FEB, pp. 439-447, 2019.

[3] M. Katsurai, "A note on keyword hierarchy construction using visual features and its application to image annotation," Ieice Technical Report Image Engineering, vol. 110, pp. 69-72, 2011.

[4] Y. H. Lu and E. Iii, "An overview of problems in image-based location awareness and navigation," Proceedings of SPIE-The International Society for Optical Engineering, vol. 5308, pp. 102-109, 2004.

[5] Z. Tian, "Dynamic visual communication image framing of graphic design in virtual reality environment," IEEE Access, vol. 99, p. 1, 2020.

[6] C.-M. Kuo, C.-K. Chang, N.-C. Yang, C.-M. Kuo, and Y.-M. Chen, "Image categorization using macro and micro sense visual vocabulary," Intelligent Data analysis and its Applications, Volume I, vol. 297, pp. 123-131, 2014.

[7] J. Fiaidhi, "Virtual scenebean: a learning object model for collaborative virtual learning environment," Informatics in Education, vol. 3, no. 2, pp. 191-218, 2004.

[8] O. O. Smolina, "Tree shaping in visual communication of urban architecture," Vestnik Tomskogo gosudarstvennogo arkhitekturno-stroitel'nogo universiteta. JOURNAL of Construction and Architecture, vol. 22, no. 1, pp. 53-62, 2020. 
[9] X. Wu, D. Chen, C.-N. Yang, and Y.-Y. Yang, “A (k,n) threshold partial reversible AMBTC-based visual cryptography using one reference image," Journal of Visual Communication and Image Representation, vol. 59, no. FEB, pp. 550-562, 2019.

[10] W. Zhang, L. Nie, and J. He, "Developer social networks in software engineering: construction, analysis, and applications," Science China, vol. 12, p. 121101, 2014.

[11] A. Z. Sampaio, D. P. Rosario, A. R. Gomes, and J. Santos, "Virtual reality applied on civil engineering education: construction activity supported on interactive models," International Journal of Engineering Education, vol. 29, no. 6, pp. 1331-1347, 2013.

[12] G. Feng, L. Ma, and X. Tan, "Visual map construction using RGB-D sensors for image-based localization in indoor environments," Journal of Sensors, vol. 2017, pp. 1-18, 2017.

[13] W. Wang, F. Gu, Z. Deng, and Y. Zhu, "Multilayer surface construction for enhancing barrier properties of cellulosebased packaging," Carbohydrate Polymers, vol. 255, no. 4, Article ID 117431, 2020.

[14] H. Wu, G. Q. Shen, X. Lin, M. Li, and C. Z. Li, "A transformerbased deep learning model for recognizing communicationoriented entities from patents of ICT in construction," $A u$ tomation in Construction, vol. 125, no. May 2021, Article ID 103608, 2021. 